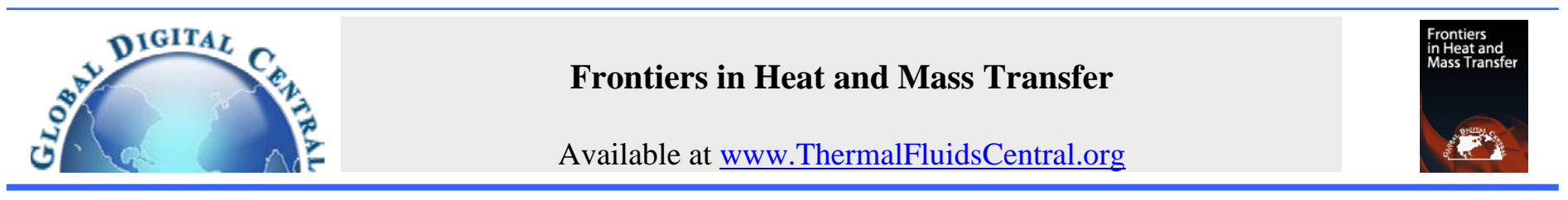

\title{
EFFECT OF MAGNETIC FIELD ON INDIRECT NATURAL CONVECTION FLOW ABOVE A HORIZONTAL HOT FLAT PLATE
}

\author{
Tapas Ray Mahapatra ${ }^{\mathrm{a}}$, Sumanta Sidui ${ }^{\mathrm{b}}$, Samir Kumar Nandy ${ }^{\mathrm{c}^{*}}$ \\ ${ }^{a}$ Department of Mathematics, Visva-Bharati, Santiniketan - 731 235, India \\ ${ }^{b}$ Department of Mathematics, Ajhapur High School, Burdwan - 713 401, India \\ ${ }^{c}$ Department of Mathematics, A.K.P.C Mahavidyalaya, Hooghly - 712 611, India
}

\begin{abstract}
The effect of variable transverse magnetic field on steady two-dimensional indirect natural convection flow of an incompressible viscous fluid over a horizontal hot flat plate is theoretically studied. The governing partial differential equations are transformed into ordinary ones by similarity transformation and solved numerically using fourth order Runge-Kutta method with shooting technique. The results are obtained for the skin friction coefficient and the local Nusselt number as well as the dimensionless velocities, temperature for some values of the magnetic parameter (M) subject to either prescribed (constant or variable) surface temperature or prescribed (variable) heat flux. It is seen that the skin friction coefficient decreases with increase in $\mathrm{M}$ for all the cases.

Keywords: Steady flow; Magnetic field; Indirect natural convection; Variable Heat Flux.
\end{abstract}

\section{INTRODUCTION}

Flow past a hot vertical plate adjacent to a viscous fluid at a lower temperature is a simple example of natural convection flow. In this case the convection takes place in boundary layer originating at the lower edge of the plate. Heat transferred from the plate to the fluid leads to an increase in temperature of the fluid near the wall causing decrease in density there and gaining buoyancy fluid moves upwards along the plate. The free convection of heat from a heated vertical plate in a fluid has been extensible studied for many years. Squire (1953) gave a review of the work. Subsequently Ostrach (1953) studied numerical solution for the free convection flow around a heated vertical plate for a wide range of values of Prandtl number.

However if the plate is horizontal the buoyancy has no component along its length and if the boundary layer exist it must be of a different kind. Natural convection flow of this different character can occur over a horizontal semi-infinite plate facing upward with temperature $\left(T_{W}\right)$ higher than that $\left(T_{\infty}\right)$ of the surrounding fluid (see Fig.1). In front of the plate, the temperature of the fluid is $T_{\infty}$ everywhere so that in this static field, there is a pressure distribution $p$, satisfying, $\partial p / \partial y=\rho_{\infty} g$ where $\rho_{\infty}$ is the density of the fluid in this region. As heat is transferred from the plate to the fluid, the fluid temperature is larger than $T_{\infty}$ above the plate and so the density $(\rho)$ is less than $\rho_{\infty}$. The reduced pressure gradient $|\partial p / \partial y|=\rho g<\rho_{\infty} g$ gives rise to reduced pressure close to the plate. Thus there is a pressure drop along the plate which is taken as the $\mathrm{x}$ direction. Due to this induced pressure gradient in the x-direction fluid flows parallel to the plate. This flow has a boundary layer character at large Grashof number and is known as indirect natural convection and was first studied by Stewartson (1958). The same problem was revisited by Gill et al. (1965). He pointed out that Stewartson's conclusion that boundary layer solution exist only when the heated plate faces downward is erroneous. They showed that boundary layer solution exists only when the heated plate faces upward and gave the correct solution to the problem. On the other hand, Wickern (see in Schlichting (2000)) investigated natural convection flow over a horizontal plate subject to uniform heat flux.

There are many studies about natural convection flow caused by immersing a hot surface in a fluid saturated porous medium at constant ambient temperature. Chamkha (2003) analyzed the heat and mass transfer laminar boundary layer flow in the presence of heat generation/absorption. Later, Chen (2004) investigated the heat and mass transfer effects of an electrically conducting fluid in magneto hydrodynamic natural convection adjacent to vertical surface. Ahmed (2010) studied the effects of chemical reaction and viscous dissipation on unsteady heat and mass transfer along an infinite vertical porous plate in the presence of magnetic field. On the other hand, Kim (2000) studied MHD natural convection flow past a moving vertical plate embedded in a porous medium. Siddiqa et al. (2010) investigated laminar natural convection flow of a viscous fluid over a semi-infinite flat plate inclined at a small angle to the horizontal. The natural convection boundary layer flow on a vertical surface in a porous medium with prescribed constant surface heat flux was considered by Merkin (2012). Very recently, a boundary layer analysis was performed for the steady laminar natural convection of a electrically conducting viscous incompressible fluid above a horizontal plate in the presence of a transverse magnetic field by Samanta and Guha (2014).

In this paper we analyze the effect of variable transverse magnetic field on the steady two-dimensional indirect natural convection flow of an incompressible viscous fluid over a hot horizontal plate which is subject to either prescribed (constant or variable) surface temperature or prescribed (variable) heat flux.

\section{FLOW ANALYSIS}

A sketch of the physical problem already described in the above introduction is given in Fig. 1 , where the $x$-axis is taken along the plate with the origin at the front end of the plate and $y$-axis is perpendicular

\footnotetext{
*Corresponding author. Email: nandysamir@yahoo.com
} 
to the plate upward. Using boundary layer approximations, the governing Magneto-Hydro-Dynamics (MHD) equations for indirect natural convection flow are as follows:

The equation of continuity is

$$
\frac{\partial u}{\partial x}+\frac{\partial v}{\partial y}=0
$$

where $(u, v)$ are the velocity components in $x$ and $y$ directions, respectively.

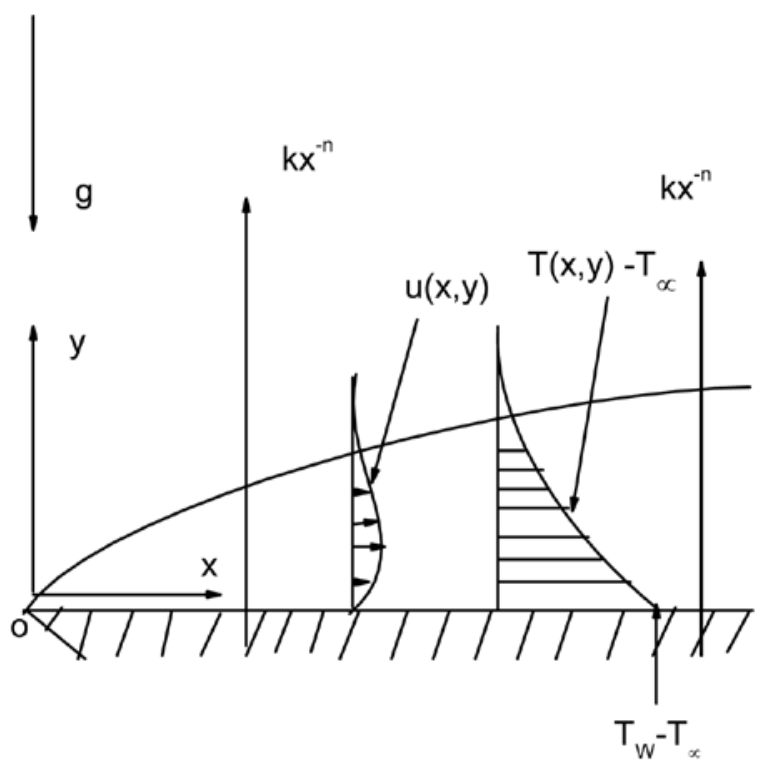

\section{Pressure drop \\ (In the boundary layer)}

Fig 1. Indirect natural convection. Formation of a pressure gradient $\partial p / \partial x$ in the boundary layer via reduced static pressure above the horizontal hot plate.

The steady two-dimensional $u$-momentum and $v$-momentum equations for the flow in the presence of variable transverse magnetic field are given by

$$
\begin{aligned}
& u \frac{\partial u}{\partial x}+v \frac{\partial u}{\partial y}=-\frac{1}{\rho_{\infty}} \frac{\partial p}{\partial x}+v_{\infty} \frac{\partial^{2} u}{\partial y^{2}}+\frac{(\boldsymbol{J} \times \boldsymbol{B})_{x}}{\rho_{\infty}} \\
& 0=-\frac{1}{\rho_{\infty}} \frac{\partial p}{\partial y}+g \beta_{\infty}\left(T-T_{\infty}\right)
\end{aligned}
$$

where $T$ denotes the temperature, $p$ is the pressure, $\rho_{\infty}$ is the fluid density at the temperature $T_{\infty}, \beta_{\infty}$ is the coefficient of thermal expansion at temperature $T_{\infty}$ and $v_{\infty}\left(=\mu / \rho_{\infty}\right)$ is the kinematic viscosity coefficient of the fluid. In writing (3), Boussinesq approximation is made so that density variation is taken into account in the buoyancy force term only. The last term in equation (2) stands for the x-component of the Lorentz force per unit mass where $\boldsymbol{J}$ is the electric current density vector and $\boldsymbol{B}(=(0, H(x), 0))$ is the imposed magnetic field. Here $H(x)\left(=k x^{Q}\right)$ denotes the variable applied transverse magnetic field where $k$ and $Q$ are constants. The value of $Q$ is determined later. In equation (2) it is assumed that the induced magnetic field is negligible in comparison to the applied magnetic field. This is a valid assumption for flow at small magnetic Reynolds number. This assumption is justified for flow of electrically conducting fluids such as liquid metals e.g. mercury, liquid sodium (see Shercliff (1995), Mahapatra et al. (2011)). It is also assumed that the external electric field is zero and the electric field due to the polarization of charges is negligible. Here the electric current flows parallel to the $z$ axis which is normal to $x-y$ plane. So by Ohm's law

$$
J_{x}=0, J_{y}=0, J_{z}=\sigma u H(x)
$$

where $\sigma$ is electrical conductivity of the fluid, which is assumed constant.

$$
\text { Using equation (4), equation (2) becomes }
$$

$u \frac{\partial u}{\partial x}+v \frac{\partial u}{\partial y}=-\frac{1}{\rho_{\infty}} \frac{\partial p}{\partial x}+v_{\infty} \frac{\partial^{2} u}{\partial y^{2}}-\frac{\sigma H^{2}(x)}{\rho_{\infty}} u$ is given by

The energy equation, neglecting viscous and Ohmic dissipation,

$$
u \frac{\partial T}{\partial x}+v \frac{\partial T}{\partial y}=a_{\infty} \frac{\partial^{2} T}{\partial y^{2}}
$$

where $a_{\infty}$ denotes the thermal diffusivity of the fluid. We now introduce the dimensionless quantities (see Schlichting and Gersten (2000))

$$
\begin{aligned}
& x^{*}=\frac{x}{l}, \bar{y}=\frac{y}{l}(G r)^{1 / 5}, \bar{u}=\frac{u}{V_{I N}}, \bar{v}=\frac{v}{V_{I N}}(G r)^{1 / 5} \\
& \bar{p}=\frac{p}{\rho_{\infty} V_{I N}{ }^{2}}, G r=\frac{g l^{3} \beta_{\infty} B}{v_{\infty}{ }^{2}}, V_{I N}=\left(g l^{1 / 2} v_{\infty}{ }^{1 / 2} \beta_{\infty} B\right)^{2 / 5}
\end{aligned}
$$

Here $l$ is a characteristic length scale, $V_{I N}$ is the characteristic velocity for indirect natural convection, $G r$ is the Grashof number, $B$ is a positive constant. In CST (Constant Surface Temperature) case $B=\Delta T(=$ $T_{W}-T_{\infty}$ ) when $T_{W}$ is greater than $T_{\infty}$. In PST (Prescribed Surface Temperature) case $B=A$; a positive constant which will be discussed in the section 3.2 and also in PHF (Prescribed Heat Flux) case $B=$ $D c_{2} / k_{\infty}$, another positive constant and $c_{2}$ is arbitrary and positive constant and $k_{\infty}$ is the coefficient of thermal conductivity and this case will be discussed in the section 3.3.

Using non-dimensional quantities (7) in equations (1),(5), (3) and (6), the boundary layer equations for indirect natural convection are

$\frac{\partial \bar{u}}{\partial x^{*}}+\frac{\partial \bar{v}}{\partial \bar{y}}=0$

$\bar{u} \frac{\partial \bar{u}}{\partial x^{*}}+\bar{v} \frac{\partial \bar{u}}{\partial \bar{y}}=-\frac{\partial \bar{p}}{\partial x^{*}}+\frac{\partial^{2} \bar{u}}{\partial \bar{y}^{2}}-\left(x^{*}\right)^{2 Q} M \bar{u}$

$\frac{\partial \bar{p}}{\partial \bar{y}}=\frac{T-T_{\infty}}{B}$

$\bar{u} \frac{\partial T}{\partial x^{*}}+\bar{v} \frac{\partial T}{\partial \bar{y}}=\frac{1}{\operatorname{Pr}} \frac{\partial^{2} T}{\partial \bar{y}^{2}}$

where $M\left(=\sigma k^{2} l^{1-2 n} / \rho_{\infty} V_{I N}\right)$ is the Hartmann number and $\operatorname{Pr}\left(=v_{\infty} / a_{\infty}\right)$ is the Prandtl number. Note that the powers in equation (7) are chosen in such a way that the following remain the same after the transformation in the limit $G r \rightarrow \infty$ : the continuity equation, a viscous term in the $x$-momentum equation as well as the pressure and buoyancy terms in the $y$-momentum equation.

Introduce the dimensionless temperature $\theta$ as

$\theta(\eta)=\frac{T-T_{\infty}}{T_{w}-T_{\infty}}, \quad$ for CST case

$\theta(\eta)=\frac{T-T_{\infty}}{B x^{* s}}, \quad$ for PST and PHF cases,

where $s$ is the wall temperature parameter. In the CST case $s=0$ and in other two cases $s \neq 0$.

Introducing the dimensionless stream function and the similarity transformations

$\bar{\psi}=c_{1} x^{* m} f(\eta), \bar{y}=c_{2} x^{* n} \eta, p=c_{3} x^{*(n+s)} \mathrm{g}(\eta)$

where $m$ and $n$ are constants, we find that (8) is identically satisfied and from the equations (9) - (11), we obtain

$$
\begin{aligned}
& \qquad \frac{c_{1}}{c_{2}^{3}} f^{\prime \prime \prime}+\frac{c_{1}^{2}}{c_{2}^{2}}\left[m f f^{\prime \prime}-(m-n) f^{\prime^{2}}\right] \\
& =c_{3}\left[(n+s) \mathrm{g}-n \eta \mathrm{g}^{\prime}\right]+\frac{c_{1}}{c_{2}} M f^{\prime} \\
& \mathrm{g}^{\prime}=\frac{c_{2}}{c_{3}} \theta
\end{aligned}
$$


$\frac{1}{c_{2}^{2}} \theta^{\prime \prime}+\frac{c_{1}}{c_{2}} \operatorname{Pr}\left[m f \theta^{\prime}-s \theta f^{\prime}\right]=0$

It is observed that for the existence of similarity solutions, we must have $m-4 n=2 m-3 n-1=s-1$ and $\mathrm{Q}=-n$. Here a prime denotes differentiation with respect to $\eta$. It is clear from above that the velocity and temperature distributions depend on the dimensionless parameters Hartmann number $M$ and Prandtl number Pr. Equations (15), (16) and (17) subject to the suitable boundary conditions are solved numerically by an efficient shooting method for different values of the parameter $M$. We first, eliminate $\theta$ between (16) and (17), the resulting equation and equation (15) are written as a system of six first order ordinary differential equations, which are solved by means of a standard fourthorder Runge-Kutta integration technique. Then a Newton iteration procedure is employed to assure quadratic convergence of the iterations required to satisfy outer boundary conditions. The constants $c_{1}$ and $c_{2}$ are arbitrary and positive. In Stewartson's (1958) notation $c_{1}=c_{2}=c_{3}=1$ and in the notation of Gill et al. (1965), $c_{2}=c_{3}$. In this paper all computations are based on $c_{1}=c_{2}=c_{3}=1$ except when we compare our results (see Table 1 ) with the corresponding results computed by Stewartson (1958) and Gill et al. (1965).

$$
\begin{aligned}
& N u(x)=\frac{q_{w}(x) l}{\lambda_{\infty}\left(T_{w}-T_{\infty}\right)} \\
& =-\frac{1}{c_{2}} \theta^{\prime}(0)\left(x^{*}\right)^{-n}(G r)^{1 / 5}, \text { for CST case } \\
& =-\frac{1}{c_{2}} \theta^{\prime}(0)\left(x^{*}\right)^{-n}(G r)^{1 / 5} \text {, for PST case } \\
& =\frac{1}{c_{2}}\left(x^{*}\right)^{-n}(G r)^{1 / 5}, \quad \text { for PHF case }
\end{aligned}
$$$$
\text { The Nusselt number at the wall is given by }
$$

where $\lambda_{\infty}$ is the thermal conductivity and $n=(2-s) / 5$. For the CST case $s=0$ and for the PHF case $\theta^{\prime}(0)=-1$.

\section{Results and discussion}

Three different cases are considered.

\subsection{Constant surface temperature (CST case)}

In this case, the boundary conditions are

$u=v=0, T=T_{w}$ at $y=0$

$u=u_{\infty}=0, p=0, T \rightarrow T_{\infty}$ as $y \rightarrow \infty$

where $T_{w}$ and $T_{\infty}$ are constants with $T_{w}>T_{\infty}$. The boundary conditions for $f$ and g are derived from (7), (12), (14), (16) and (21) as

$f(0)=0, f^{\prime}(0)=0, g^{\prime}(0)=\frac{c_{2}}{c_{3}}$

$f^{\prime}(\infty)=0, g(\infty)=0, g^{\prime}(\infty)=0$ $\mathrm{s}=0$ as

The equations of $f, g$ and $\theta$ are obtained from (15)-(17) by putting

$$
\begin{aligned}
& \quad \frac{c_{1}}{c_{2}^{3}} f^{\prime \prime \prime}+\frac{c_{1}^{2}}{c_{2}^{2}}\left[m f f^{\prime \prime}-(m-n) f^{\prime^{2}}\right] \\
& =c_{3} n\left[\mathrm{~g}-\eta \mathrm{g}^{\prime}\right]+\frac{c_{1}}{c_{2}} M f^{\prime} \\
& \mathrm{g}^{\prime}=\frac{c_{2}}{c_{3}} \theta \\
& \frac{1}{c_{2}^{2}} \theta^{\prime \prime}+\frac{c_{1}}{c_{2}} \operatorname{Pr} m f \theta^{\prime}=0
\end{aligned}
$$

with the condition $m-4 n=2 m-3 n-1=-1$.
Equation (24) - (26) are solved numerically subject to the boundary condition (22) and (23) using the technique as stated in earlier section.

Table 1 gives the comparison of the values of $f^{\prime \prime}(0),-\theta^{\prime}(0),-\mathrm{g}(0)$ and $f(\infty)$ calculated in the present study (A) with those of Stewartson (1958) (B) and Gill et al. (1965) (C) when $c_{1}=c_{2}=$ $c_{3}=1, M=0$ and $\operatorname{Pr}=0.72$. A good agreement in the values is observed.

Table 1: Comparison of the values of $f^{\prime \prime}(0),-\theta^{\prime}(0),-\mathrm{g}(0)$ and $f(\infty)$ of (A) with those of (B) and (C) where $M=0, \operatorname{Pr}=0.72$

\begin{tabular}{|l|l|l|l|c|}
\hline & $f^{\prime \prime}(0)$ & $-\theta^{\prime}(0)$ & $-g(0)$ & $f(\infty)$ \\
\hline A & 0.9784 & 0.3574 & 1.7349 & 2.3301 \\
\hline B & 0.9710 & 0.3580 & 1.7300 & 2.3000 \\
\hline C & 0.9787 & 0.3574 & ---- & ---- \\
\hline
\end{tabular}

Figure 2 shows the variation of horizontal velocity for different values of $M$ where $\operatorname{Pr}=0.72$. It is observed that the velocity parallel to the plate decreases with increase in $M$ up to a certain distance from the plate but beyond that distance opposite trend is observed. Variation of wall temperature for several values of $M$ are presented in the Fig. 3 for $\operatorname{Pr}=0.72$. It shows that the temperature at a point increases with increase in $M$. Fig. 4 represents the variation of normal velocity to the plate for several values of $M$ when $\operatorname{Pr}=0.72$. It is observed that up to a small distance from the plate the normal velocity increases with increase in $M$ but beyond that distance opposite trend is observed.

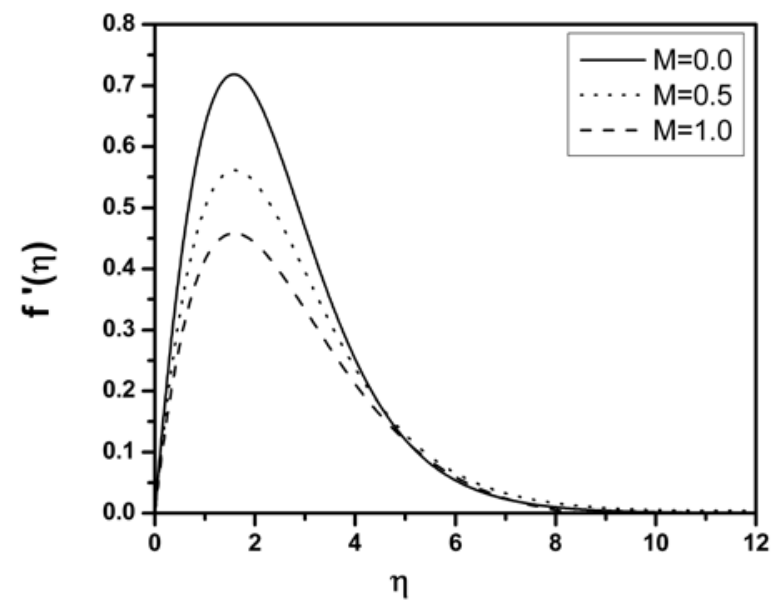

Fig. 2 Variation of $f^{\prime}(\eta)$ for several values of $M$ for CST case with $\operatorname{Pr}=0.72$.

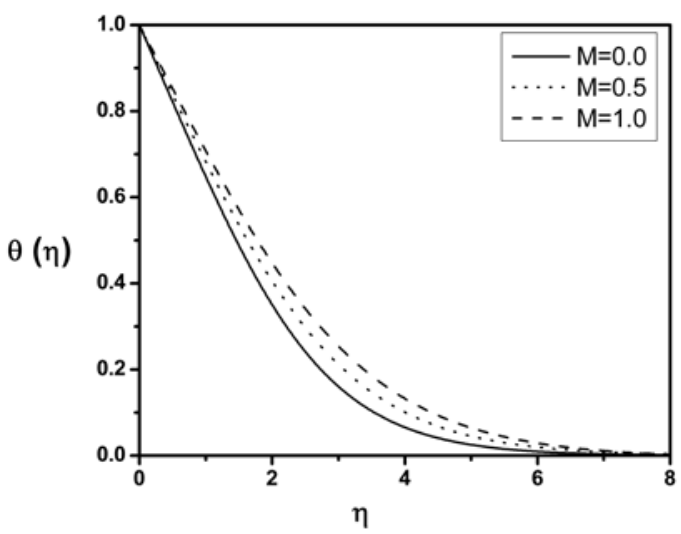

Fig. 3 Variation of temperature profile $\theta(\eta)$ for several values of $M$ for CST case with $\operatorname{Pr}=0.72$. 


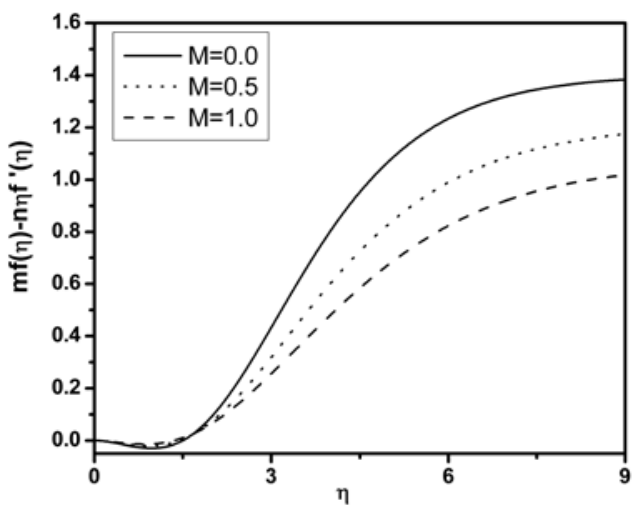

Fig. 4 Variation of $m f(\eta)-n \eta f^{\prime}(\eta)$ velocity normal to the plate for several values of $M$ for the CST case with $\operatorname{Pr}=0.72$.

Table 2: Values of $f^{\prime \prime}(0),-\theta^{\prime}(0)$ for several values of $M$ in CST case when $\operatorname{Pr}=0.72$

\begin{tabular}{|c|c|c|c|}
\hline $\mathrm{M}$ & 0.0 & 0.5 & 1.0 \\
\hline$f^{\prime \prime}(0)$ & 0.9784 & 0.8321 & 0.7487 \\
\hline$-\theta^{\prime}(0)$ & 0.3574 & 0.3212 & 0.2957 \\
\hline
\end{tabular}

Table 2 gives the values of wall shear stress $\left(f^{\prime \prime}(0)\right)$ and surface heat flux $\left(-\theta^{\prime}(0)\right)$ for several values of $M$, when $\operatorname{Pr}=0.72$ for CST case. It can be seen from Table 2 that the surface shear stress decreases with increase in $M$. It is seen that the surface heat flux $-\theta^{\prime}(0)$ also decreases with increase in $M$.

\subsection{Prescribed surface temperature (PST case)}

Here the boundary conditions are

$u=v=0, T=T_{w}\left(=T_{\infty}+A x^{* s}\right)$ at $y=0$

$u=u_{\infty}=0, p=0, T \rightarrow T_{\infty}$ as $y \rightarrow \infty$

where $T_{W}>T_{\infty}$. Note that the temperature parameter $s$ is introduced earlier is the index of power law variation of surface temperature shown above. Further the constant B in equation (7) that remained unspecified so far is now A, which is a positive constant. The boundary conditions for $f$ and $g$ are derived from (7), (13), (14), (16) and (27) as

$f(0)=0, f^{\prime}(0)=0, \mathrm{~g}^{\prime}(0)=\frac{c_{2}}{c_{3}}$
$f^{\prime}(\infty)=0, \mathrm{~g}(\infty)=0, \mathrm{~g}^{\prime}(\infty)=0$

In this case the equations of $f, g$ and $\theta$ are (15), (16) and (17) with the condition $m-4 n=2 m-3 n-1=s-1$. It is clear from equations (15), (16) and (17) that the velocity and temperature distribution depend on the dimensionless parameter Hartmann number $M$, Prandtl number $\mathrm{Pr}$ and wall temperature parameter s. Equations (15), (16) and (17) subject to the boundary conditions (28) and (29) are solved numerically by using the same method which is used in CST case.

Figure 5 represents the variation of horizontal velocity for different values of $M$ where $\operatorname{Pr}=0.72$ and $s=0.3$. It shows that the velocity parallel to the plate decreases with increase in $M$ for fixed values of $P r$ and $s$. Variation of temperature are presented in Fig.6 for several values of $M$ where $P r=0.72$ and $s=0.3$. It is observed that for fixed value of $P r$ and $s$, temperature at a point increases with increase in $M$. Fig.7 shows the variation of normal velocity with $M$ where $P r=0.72$ and $s=0.3$. It shows that this velocity increases with increase in $M$ up to a small distance from the plate but beyond that opposite trend is observed.

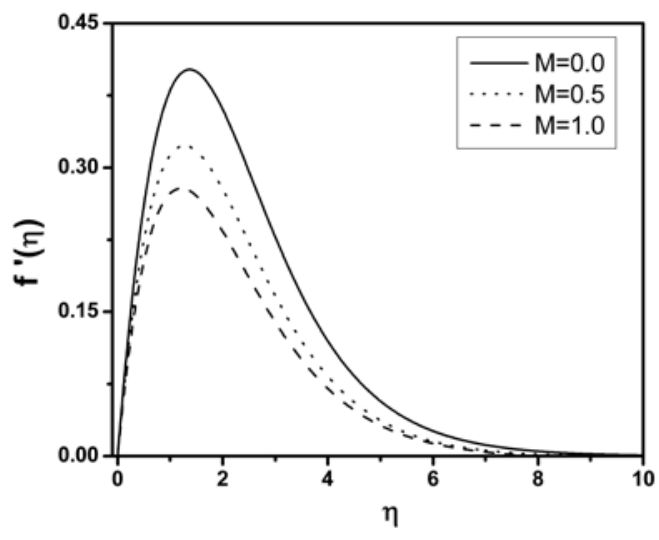

Fig. 5 Variation of $f^{\prime}(\eta)$ for several values of $M$ for PST case with $\operatorname{Pr}=2.0$ and $\mathrm{s}=0.3$.

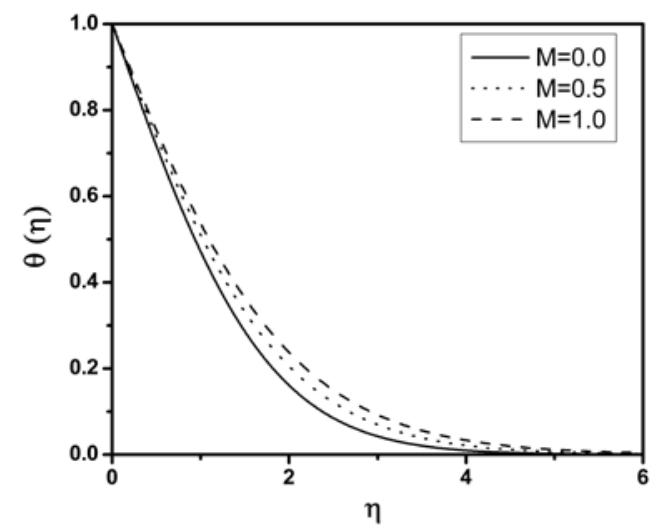

Fig. 6 Variation of temperature profile $\theta(\eta)$ for several values of $M$ for PST case with $\operatorname{Pr}=2$., $s=0.3$.

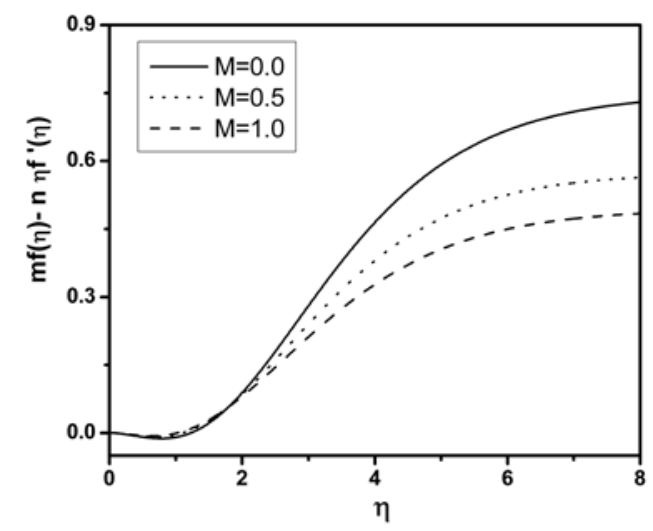

Fig. 7 Variation of $m f(\eta)-n \eta f^{\prime}(\eta)$ velocity normal to the plate for several values of $M$ for the CST case with $\operatorname{Pr}=2.0$ and $\mathrm{s}=0.03$.

Table 3: Values of $f^{\prime \prime}(0),-\theta^{\prime}(0)$ for several values of $M$ in PST case when $\operatorname{Pr}=2.0$ and $s=0.3$

\begin{tabular}{|c|c|c|c|}
\hline $\mathrm{M}$ & 0.0 & 0.5 & 1.0 \\
\hline$f^{\prime \prime}(0)$ & 0.6924 & 0.6294 & 0.5925 \\
\hline$-\theta^{\prime}(0)$ & 0.5832 & 0.5374 & 0.5066 \\
\hline
\end{tabular}


Table 3 gives the values of $f^{\prime \prime}(0)$ and $-\theta^{\prime}(0)$ for several values of $M$ when $\operatorname{Pr}(=2.0)$ and $s(=0.3)$ are kept fixed. It can be seen from Table 3 that the surface shear stress decreases with increase in $M$ for fixed values of $s$ and Pr. It can also be seen from Table 3 that the surface heat flux $-\theta^{\prime}(0)$ decreases with increase in $M$ for any fixed value of $s(=$ $0.3)$ and $\operatorname{Pr}(=2.0)$.

When $s=-m$, i.e., when $s=-0.5$, the energy equation (17) becomes

$$
\theta^{\prime \prime}+c_{1} c_{2} m \operatorname{Pr}\left(f \theta^{\prime}+\theta f^{\prime}\right)=0
$$

Integrating (30) once w.r.t. $\eta$ and using the boundary condition $\theta(\infty)=$ 0 , we obtain

$\theta^{\prime}(\eta)=-c_{1} c_{2} m \operatorname{Pr} f(\eta) \theta(\eta)$.

As $f(0)=0$, equation (31) shows that $\theta^{\prime}(0)=0$ when $s=-0.5$. It is interesting to note that wall heat flux vanishes when the surface temperature varies as $x^{-1 / 2}$. It may be noted that Gill et al. (1965) considered only a particular case of wall temperature variation viz., $T_{W}-$ $T_{\infty}=B x^{*-1 / 2}$.

\subsection{Prescribed power law heat flux (PHF case)}

In PHF case the dimensionless temperature variable $\theta(\eta)$ is defined as $\theta(\eta)=\frac{T(x, y)-T_{\infty}}{D x^{* s} c_{2} / k_{\infty}}$

Thus the constant $B$ which remained so far unspecified, for this case is $D c_{2} / k_{\infty}$, where $k_{\infty}$ is the thermal conductivity of the fluid and $D$ is a positive constant. In this case the corresponding boundary conditions are

$$
\begin{aligned}
& u=v=0, q_{w}=-k_{\infty}\left(\frac{\partial T}{\partial y}\right)_{w}=D\left(x^{*}\right)^{s-n} \text { at } y=0 \\
& u=u_{\infty}=0, p=0, T \rightarrow T_{\infty} \text { as } y \rightarrow \infty
\end{aligned}
$$

The boundary conditions for $f$ and $g$ are derived from (7), (14), (16), (32) and (33) as

$$
\begin{aligned}
& f(0)=0, f^{\prime}(0)=0, \mathrm{~g}^{\prime \prime}(0)=-\frac{c_{2}}{c_{3}} \\
& f^{\prime}(\infty)=0, \mathrm{~g}(\infty)=0, \mathrm{~g}^{\prime}(\infty)=0
\end{aligned}
$$

In this case the equations of $f, g$ and $\theta$ are (15), (16) and (17) with the condition $m-4 n=2 m-3 n-1=s-1$. Note that for the case of uniform heat flux, $s=n$ (see equation (33)). Then the above equation for $m, n$ and $s$ give $s=1 / 3$ which agrees with the result of Wickern (2000).

Here the velocity and temperature distributions depend on three dimensionless parameters Hartmann number $M$, Prandtl number $\mathrm{Pr}$ and wall heat flux parameter s. Equations (15), (16) and (17) subject to the boundary conditions (34) and (35) are solved numerically by using the same method as described earlier. Here, wall temperature will be different for different physical situation contrast to the cases of PST where it attains the constant value.

This behavior of the temperature profile is the consequences of prescribed boundary conditions. The qualitative behaviors of velocity, temperature and normal velocity distributions due to variation of the parameter $M$ are similar to those of the PST case.

Figure 8 shows the variation of horizontal velocity for several values of $M$. This velocity decreases with increase in $M$. Fig.9 represents the variation of temperature for several values of $M$ for $\operatorname{Pr}=0.1$ and $s=$ 0.1 . The wall temperature $\theta(0)$ increases with increase in $M$. Variation of normal velocity for several values of $M$ when $\operatorname{Pr}=0.1$ and $s=0.1$ are presented in the Fig.10. It shows that this velocity almost remains constant with increase in $M$ up to a small distance from the plate but beyond that it decreases with increase in $M$.

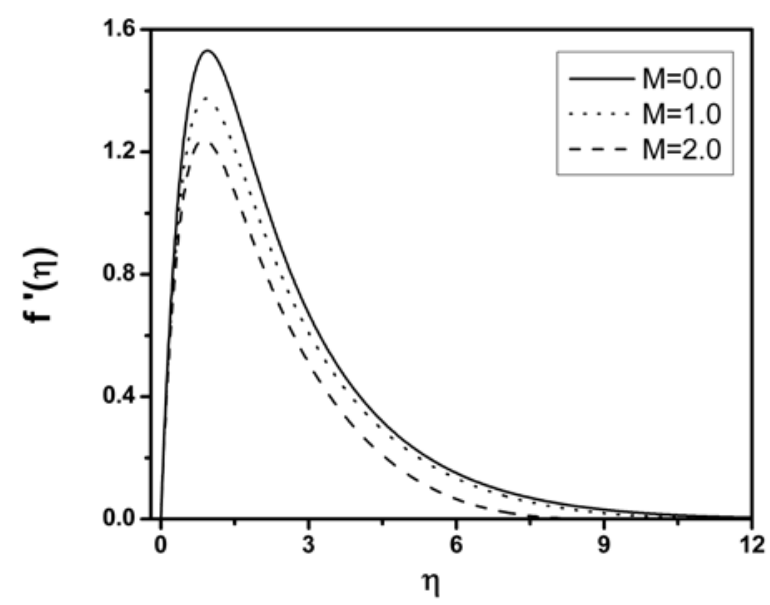

Fig. 8: Variation of $f^{\prime}(\eta)$ for several values of $M$ for PHF case with $\operatorname{Pr}=0.1$ and $s=2.0$

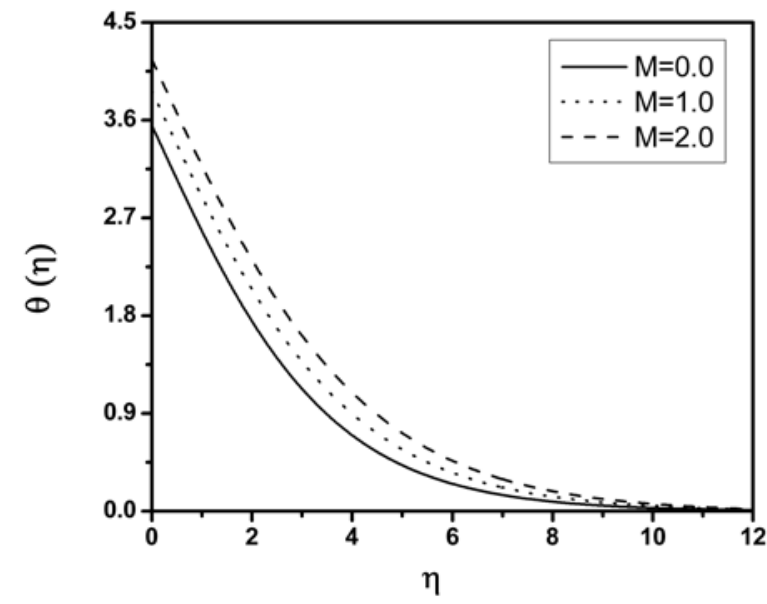

Fig. 9: Variation of temperature profile $\theta(\eta)$ for several values of $M$ for PHF case with $\operatorname{Pr}=0.1, s=0.1$.

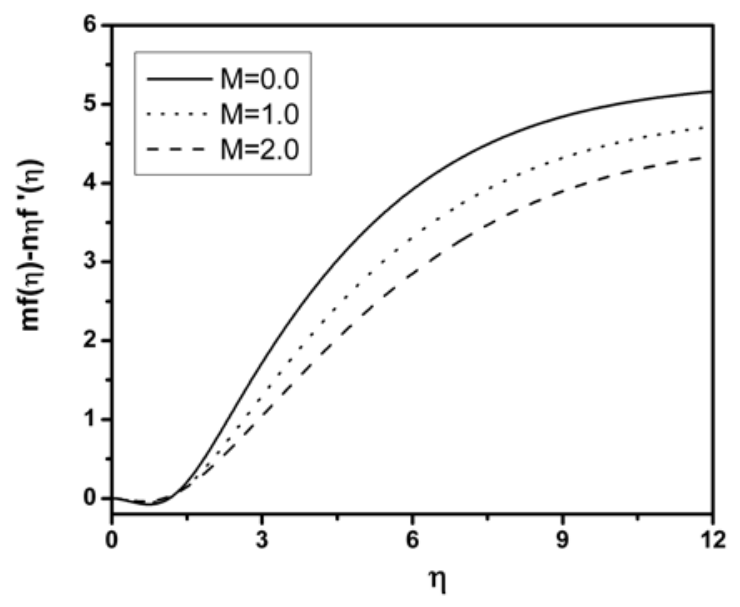

Fig. 10: Variation of $m f(\eta)-n \eta f^{\prime}(\eta)$ velocity normal to the plate for several values of $M$ for the PHF case with $\operatorname{Pr}=0.1$ and $s=0.1$. 
Table 4: Values of $f^{\prime \prime}(0)$ and $\theta(0)$ for several values of $M$ in PHF case when $\operatorname{Pr}=1.0$ and $s=2.0$

\begin{tabular}{|c|l|l|l|}
\hline $\mathrm{M}$ & 0.0 & 0.5 & 1.0 \\
\hline$f^{\prime \prime}(0)$ & 1.1989 & 1.1885 & 1.1820 \\
\hline$\theta(0)$ & 1.1884 & 1.2224 & 1.2509 \\
\hline
\end{tabular}

Table 4 gives the values of $f^{\prime \prime}(0)$ and $\theta(0)$ for several values of $M$ where $\operatorname{Pr}(=1.0)$ and $s(=2.0)$ are kept fixed. It can be seen from Table 4 that the surface shear stress decreases with increase in $M$ for any fixed value of $s$ and $P r$. It can also be seen from Table 4 that the temperature $\theta(0)$ increases with increase in $M$.

\section{Concluding Remarks}

We have obtained an exact similarity solution of the Navier-Stokes equations which represents steady two-dimensional indirect natural convection flow of an incompressible viscous fluid above a horizontal hot flat plate in the presence of a variable transverse magnetic field. Here the velocity field is dependent on the temperature field. Different boundary conditions are considered for temperature field and three different cases are examined. For all the three cases, surface shear stress decreases with increase in $M$ for fixed values of Pr and $s$, where $s$ is the parameter characterizing the wall temperature variation for PST and CST cases but wall heat flux parameter for PHF cases, Pr is the Prandtl number and $M$ is the magnetic parameter.

\section{Case 1. CST (Constant Surface Temperature):}

It is found that at first the velocity at a point parallel to the plate decreases with increase in $M$ but after a certain distance perpendicular to the sheet, opposite trend is observed. Temperature at a point increases with increase in $M$. The surface heat flux decreases with increase in $M$.

\section{Case 2. PST (Prescribed Surface Temperature):}

Similarity solutions for velocity and temperature distributions are obtained in this case. It is found that the velocity at a point parallel to the plate decreases with increase in $M$. The surface heat flux decreases with increase in $M$. For fixed values of $\operatorname{Pr}$ and $s$ temperature at a point increases with increase in the parameter $M$.

\section{Case 3. PHF (Prescribed Heat Flux):}

Qualitative behaviours of temperature distribution in this case follow the similar pattern as those for the case of PST. It is seen that for the fixed values of Pr and $s$ the wall temperature $\theta(0)$ increases with increase in $M$. It is also found that the velocity at a point parallel to the plate decreases with increase in $M$.

\section{Acknowledgements}

The work of one of the authors (T.R.M.) is supported under SAP (DRS Phase-III) under UGC, New Delhi, India. The authors thank the referees for their critical comments which enabled an improved presentation of the paper.

\section{References}

Squire, H.B., 1953, "Modern Developments in Fluid Dynamics: High Speed Flow," ed. L. Howarth; Oxford, Chap. 10. http://dx.doi.org/10.1002/zamm.19540341215
Ostrach, S., 1953, “An Analysis of Laminar Free-Convection Flow and Heat Transfer about a Flat Plate Parallel to the Direction of the Generating Body Force,” N.A.C.A. Rep. No.1111.

http://naca.central.cranfield.ac.uk/reports/1953/naca-report-1111.pdf

Stewartson, K., 1958, "On the Free Convection from a Horizontal Plate,” Z. Angew. Math. Phys. (ZAMP), pp. 276 - 282.

http://dx.doi.org/10.1007/BF02033031

Gill, W.N., Zeh, D.W., and Casal E.D., 1965, "Free Convection on a Horizontal Plate,” Z. Angew. Math. Phys. (ZAMP), 16, pp. 539 - 541. http://dx.doi.org/10.1007/BF01593934

Schlichting, H., 2000, Boundary-Layer Theory, 8th revised and enlarged ed., Springer-Verlag, Berlin/Heidelberg.

http://dx.doi.org/10.1007/978-3-540-95998-4_2

Chamkha, A.J., 2003, "MHD Flow of a Uniform Stretched Vertical Permeable Surface In The Presence Of Heat Generation/absorption And a Chemical Reaction,” Int. Commu. Heat Mass Trans., 30, pp. 413-422. http://dx.doi.org/10.1016/S0735-1933(03)00059-9

Chen, C.H., 2004, "Heat and Mass Transfer In MHD Flow by Natural Convection from a Permeable, Inclined Surface with Variable Wall Temperature and Concentration,” Acta Mech., 172, pp. 219-235. http://dx.doi.org/10.1007/s00707-004-0155-5

Ahmed, S., 2010, "Effect of Viscous Dissipation and Chemical Reaction on Transient Free Convective Flow over a Vertical Porous Plate," Journal of Energy Heat Mass Trans., 32, pp. 311-332.

Kim, Y.J, 2000, “Unsteady MHD Convective Heat Transfer Past a SemiInfinite Vertical Porous Moving Plate with Variable Suction,” Int. J. Eng. Sc., 38, pp. 833-845.

http://dx.doi.org/10.1016/S0020-7225(99)00063-4

Siddiqa, S., Asghar S. and Hossain M.A., 2010, "Natural Convection Flow over Inclined Flat Plate with Internal Heat Generation and Variable Viscosity," Mathematical and Computer Modelling," 52, pp. 1739-1761. http://dx.doi.org/10.1016/j.mcm.2010.07.001

Merkin, J.H., 2012, "Natural Convective Boundary Layer Flow in a Heat Generating Porous Medium with a Constant Surface Heat Flux," European Journal of Mechanics- B/Fluids, 36, pp. 75-81. http://dx.doi.org/10.1016/j.euromechflu.2012.04.004

Samanta, S., Guha A., 2014, “Analysis of Heat Transfer and Stability of Magnetohydrodynamic Natural Convection above a Horizontal Plate with Heat Flux Boundary Condition,” Int. J. Heat Mass Trans., 70, pp. 793-802.

http://dx.doi.org/10.1016/j.ijheatmasstransfer.2013.10.049

Mahapatra, T.R., Nandi, S.K., Gupta, A.S., 2011, "Momentum and Heat Transfer in MHD Stagnation-point Flow over a Shrinking Sheet," Journal of Applied Mechanics, 78, pp. 021015:1-8 http://dx.doi.org/10.1115/1.4002577

Shercliff, J.A., 1965, A Text Book of Magneto-Hydro-Dynamics, Pergamon, Oxford. 